\title{
Optimization of the high pressure leaching of Complex Copper Concentrates of Codelco using a process simulator
}

\author{
N.P. Werth \\ EcoMetales Limited, Providencia, Santiago, Chile
}

\begin{abstract}
In recent years, arsenic (As) content in copper concentrates has increased along with environmental restrictions for their transport and processing. Under this scenery is being developed the Complex Concentrate Leaching Project (PLCC), which allows the treatment of copper concentrates with high As content $(>0.5 \%)$ through a high pressure $(2873 \mathrm{kPa})$ and temperature $\left(220^{\circ} \mathrm{C}\right)$ leaching process within an autoclave. The addition of high purity oxygen and cooling water to control the temperature in the different autoclave compartments is possible to dissolve over $99 \%$ of copper and As compounds. Dissolved As is then precipitated as an arsenical residue. The PLS is sent to a solvent extraction plant to produce copper cathodes, where it is previously mixed with heap/dump PLS solutions. On the other hand, the arsenical residue is stored on a deposit or sent to a silver recovery process. The gases from the autoclave, slurry depressurization and slurry cooling are sent to a gas cleaning system, where water and entrainments are recovered before emitting the gas to the atmosphere. A process simulator was developed in order to assist the review of the feasibility study and analyze different process configurations. The purpose of this work is to describe the process simulator developed for the PLCC project and present some simulation results for different process configuration analyzed during the feasibility of the PLCC project, which permitted to improve the process and reduce the CAPEX and OPEX.
\end{abstract}

\section{INTRODUCTION}

Between years 2000 and 2015 world copper production from concentrates has increased approximately from eleven to fifteen millions of metric tonnes per year. In 2014 , Chile produced $26.5 \%$ of the total copper from concentrates and in the future it is expected that copper production in Chile will rise, mainly associated to production of concentrates (Fig. 1).

In recent years arsenic (As) content in copper concentrates has increased along with environmental restrictions for their handling, transport and processing. China has set a benchmark maximum of $0.5 \%$ for the As content in copper concentrates and in the future. It is expected that this value is going to be more restrictive.

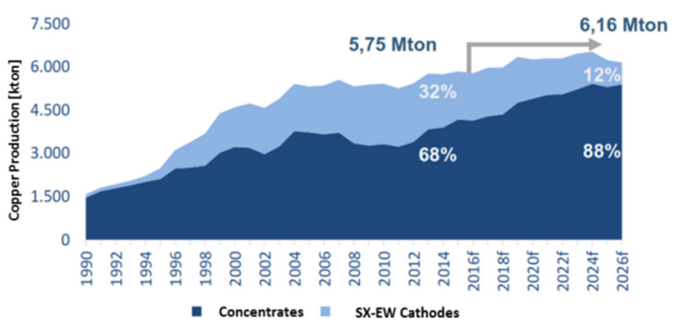

Figure 1. Copper production in Chile.
Under this scenery, the Complex Concentrate Leaching Project (PLCC) has developed leaching process using autoclave technology which allows the treatment of copper concentrates with high As content $(>0.5 \%)$ of Codelco through a high pressure $(2873$ $\mathrm{kPa})$ and temperature $\left(220^{\circ} \mathrm{C}\right)$ (Marsden \& Brewer, 2003; Parra et al., 2017).

This work describes the process simulator that was developed in order to assist the review of the feasibility study and analyze different process configurations which allowed reducing the CAPEX and OPEX of the project.

\section{METHODS/EXPERIMENTAL}

During the years 2014 and 2016, it was developed the prefeasibility and feasibility studies of the PLCC project, respectively. In both stages, batch and pilot campaigns were done in order to define the operational and design parameters for the high pressure leaching and solid/liquid separation stages (Marsden, 2007; McDonald \& Muir, 2007).

Together with these tests, a process simulator was developed using Matlab/Simulink ${ }^{\circledR}$ software including the mass and heat balance of all the stages of the process. This permitted to review the calculations done by the engineering company (Hatch) and to analyze some changes in the flowsheet in order to improve the OPEX and CAPEX of the project. 


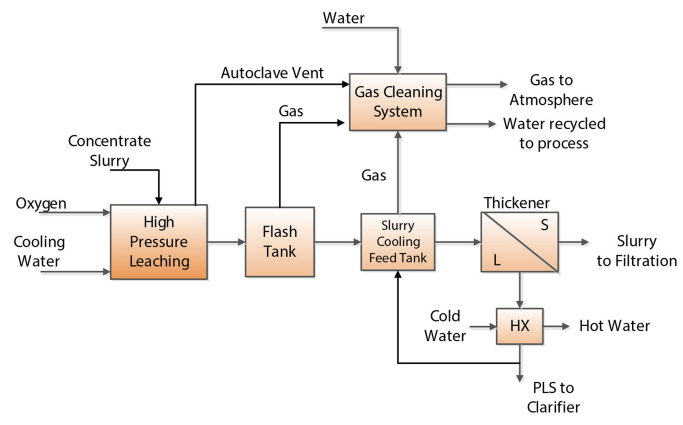

Figure 2. Prefeasibility simplified process flow diagram.

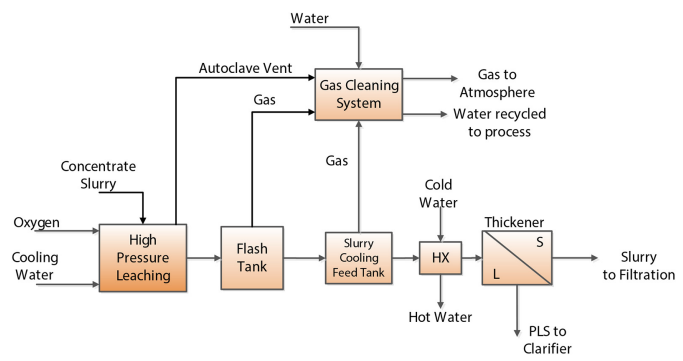

Figure 3. Feasibility simplified process flow diagram.

The chemical reactions and their extent inside the autoclave were defined and adjusted to represent the results obtained for the different processes and feed conditions. All the parameters for the rest of the processes were adjusted according to the specifications done in the engineering design.

\section{RESULTS}

During the prefeasibility, it was seen that in order to minimize the As redissolution after the high pressure leaching in the autoclave, the slurry has to be cooled to $40^{\circ} \mathrm{C}$. To do this, it was defined to recirculate part of the cooled slurry after the thickening stage to the seal tank (Figure 2). This permitted to cool the flash tank slurry from $90^{\circ} \mathrm{C}$ to $71^{\circ} \mathrm{C}$. A heat exchange stage was considered after the thickener, which allowed cooling the slurry from $69^{\circ} \mathrm{C}$ to $40^{\circ} \mathrm{C}$.
During the feasibility, another option was analyzed, which considered cooling the slurry before the thickener (Figure 3). A new simulation was done considering this new flowsheet, and it was seen that doing this modification the flowrate to the thickener was reduced $42 \%$, which allowed reducing the size of the slurry cooling feed tank and thickener. This modification permitted to reduce the CAPEX in 3 MUS\$. Also this rapid cooling allowed improving the As precipitation as ferric arsenate from $85 \%$ to $90 \%$.

Another point analyzed during the feasibility was the water make-up in the process. During the prefeasibility, it was defined to use cooling towers to cool the water used in: i) gas cleaning system, ii) slurry cooling system, and iii) oxygen plant. Most of the make-up water of the process was due to the loss of water (evaporation) in the cooling towers, so during the feasibility the use of dry coolers was analyzed, instead of cooling towers. A new simulation was done considering the use of only dry coolers and it was seen that make-up water was reduced $90 \%$. This modification allowed to

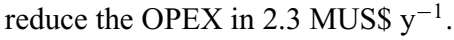

\section{CONCLUSION}

The development of a simulator for the high pressure leaching of copper concentrates with high As contents of Codelco allowed to review the engineering of the project and to analyze different process configurations, which allowed reducing the CAPEX, OPEX and improving the As precipitation.

\section{REFERENCES}

Marsden, J. \& Brewer, R. 2003. Hydrometallurgical processing of copper concentrates by Phelps Dodge at the Bagdad mine in Arizona. Proceedings of the ALTA copper conference. Melbourne, Australia 2003. ALTA Metallurgical Services.

Marsden, J. 2007. Medium-temperature pressure leaching of copper concentrates Part I: Chemistry and initial process development. Miner. Metall. Proc. 24(4): 193-204.

McDonald, R.G. \& Muir, D.M. 2007. Pressure Oxidation leaching of chalcopyrite. Part I: Comparison of high and low temperature reaction kinetics and products Hydrometallurgy 86: 191-205.

Parra, N., Acuña, M. \& Fuentes, O. 2017. PLCC Project: Autoclave Technology Applied to Complex Copper Concentrates. Hydroprocess ICMSE 2017, 9th International Seminar on Process Hydrometallurgy. Santiago, Chile. 